Recepción: 07 / 04 / 2018

Aceptación: 03 / 06 / 2018

Publicación: 02 / 07 / 2018

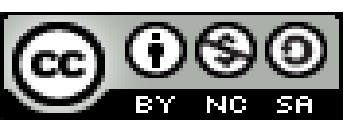

Ciencias económicas y empresariales

Artículo de Investigación

\title{
La nueva administración del siglo XXI
}

\section{The new administration of the XXI century}

\section{A nova administração do século 21}

\author{
Ibett M. Jácome-Lara ${ }^{\mathrm{I}}$ \\ ibett.jacome@utc.edu.ec \\ Marlon R. Tinajero-Jiménez II \\ marlon.tinajero@utc.edu.ec \\ Iván M. Suárez-Guevara ${ }^{\mathrm{III}}$ \\ ivan.suarez@utc.edu.ec
}

Correspondencia: ibett.jacome@utc.edu.ec

\footnotetext{
I Magister en Gestión Empresarial, Especialista en Gestión Avanzada de Operaciones, Ingeniero Comercial, Docente de la Universidad Técnica de Cotopaxi, Latacunga, Ecuador.

${ }^{\text {II }}$ Magister en Gestión de Empresas Mención Pequeñas y Medianas Empresas, Ingeniero Comercial, Docente de la Universidad Técnica de Cotopaxi, Latacunga, Ecuador.

III Magister en Gestión de Empresas Mención Pequeñas y Medianas Empresas, Ingeniero de Empresas, Docente de la Universidad Técnica de Cotopaxi, Latacunga, Ecuador.
} 


\title{
Resumen
}

Los continuos cambios del mundo organizacional representan un desafío para el éxito de las empresas, esto conlleva a que su administración tenga una estructura cada vez más flexible, que le permita adaptarse a las circunstancias actuales e incluso predecir nuevos escenarios para una gestión eficiente de cara a lo que resta de siglo XXI. El presente trabajo, plasma una serie de conceptos, opiniones y análisis relacionados con el modelo de administración para el futuro. El mismo se realizó mediante una revisión de tipo documental por la naturaleza de sus fuentes, en su mayoría digitales. Las empresas deben adquirir las estrategias necesarias ante los constantes cambios del sistema, el estudio de los retos que se le presenta a la administración del siglo XXI es fundamental para el nuevo modelo administrativo. Elementos como la globalización, la automatización de los procesos, el desarrollo organizacional, entre otros, son vitales para las proyecciones de sus características fundamentales. La transformación de la administración es imperiosa para garantizar el alcance de las metas y la permanencia de su efectividad y eficiencia en el futuro.

Palabras clave: nueva; administración; siglo; modelo; transformación.

\begin{abstract}
The continuous changes of the organizational world represent a challenge for the success of the companies, this entails that their administration has an increasingly flexible structure, which allows it to adapt to current circumstances and even predict new scenarios for an efficient management in order to what remains of the 21st century. The present work captures a series of concepts, opinions and analyzes related to the management model for the future. It was carried out through a documentary type review due to the nature of its sources, mostly digital. Companies must acquire the necessary strategies in the face of constant changes in the system, the study of the challenges presented to the administration of the 21 st century is fundamental for the new administrative model. Elements such as globalization, the automation of processes, organizational development, among others, are vital for the projections of their fundamental characteristics. The transformation of the administration is imperative to guarantee the reach of the goals and the permanence of its effectiveness and efficiency in the future.
\end{abstract}

Keywords: new; administration; century; model; transformation. 


\section{Resumo}

As mudanças contínuas do mundo organizacional representam um desafio para o sucesso das empresas, o que implica que sua administração tem uma estrutura cada vez mais flexível, que permite adaptar-se às circunstâncias atuais e até prever novos cenários para uma gestão eficiente, a fim de o que resta do século 21 . O presente trabalho captura uma série de conceitos, opiniões e análises relacionadas ao modelo de gestão para o futuro. Foi realizado através de uma revisão do tipo documental, devido à natureza de suas fontes, principalmente digital. As empresas devem adquirir as estratégias necessárias diante de constantes mudanças no sistema, o estudo dos desafios apresentados à administração do século XXI é fundamental para o novo modelo administrativo. Elementos como globalização, automação de processos, desenvolvimento organizacional, entre outros, são vitais para as projeções de suas características fundamentais. A transformação da administração é imperativa para garantir o alcance dos objetivos e a permanência de sua eficácia e eficiência no futuro.

Palavras chave: novo; administração; século; modelo; transformação.

\section{Introducción}

La administración es muy antigua, y está relacionada con el hombre desde que éste usó el razonamiento, ya que se buscó la manera de delimitar tareas, tener un líder, tomar decisiones, planear y llevar a cabo acciones encaminadas a lograr algún objetivo tanto social como individual. El reconocimiento como ciencia de la Administración se viene a dar mucho tiempo después, aunque siempre a lo largo de la evolución historia del hombre día a día existe la necesidad de planificar, organizar, dirigir y controlar que siempre han estado, pero que han sido enfocadas de maneras distintas, ya que cada pueblo o sociedad fue aplicándolas según las necesidades y contextos que se presentaban en esos momentos razón por la que han ido evolucionando las formas de administrar. Hoy en día herramientas de desarrollo, innovación, gestión, creatividad, competencias, procesos, estrategias han sido implementadas para mejorar la administración y optimizar los tiempos y recursos para obtener el mejor beneficio de ellos y alcanzar los objetivos. (Villarreal, 2013)

Chiavenato (2004), define la administración, como "el proceso de planear, organizar, dirigir y controlar el uso de los recursos para logar los objetivos organizacionales". (p. 10) 
En tal sentido, podemos ver, de manera general, como la administración de empresas es un proceso dirigido a cumplir las metas organizacionales.

El problema se presenta ante los constantes cambios que surgen con el avance del tiempo, lo que convierte a la empresa en una entidad cambiante y adaptable a las circunstancias que la rodean. En el sistema actual, de cara al siglo XXI, esta teoría plantea una interrogante: ¿qué hacer para administrar de la mejor manera?

Se requieren estudios teóricos, que permitan a las organizaciones analizar los cambios inminentes y su correcta adaptación a los mismos, con la finalidad de enfrentar de manera victoriosa los retos del siglo XXI, el presente trabajo es esa herramienta que aporta un enfoque de la visión de la nueva administración, de cómo se están y se estima, se presentarán los diferentes retos que las organizaciones deben asumir y confrontar, así como las principales características que las empresas deben adoptar de cara al futuro y en pro de la obtención de los mejores resultados en el alcance de sus objetivos.

\section{Materiales y métodos}

El presente estudio se centró en desarrollar aspectos relacionados con la administración del siglo XXI, específicamente los retos que enfrentará dicha disciplina de cara al futuro y las características que las empresas deben adoptar para enfrentar tales desafíos, todo ello con la finalidad de plasmar en base a opiniones variadas de expertos del tema y concluir un análisis crítico de la posición que deben asumir las empresas del siglo XXI para adaptarse a los cambios inminentes del mundo organizacional.

El presente estudio se realizó a través de una investigación de tipo documental, sustentada por fuentes digitales, las cuales se recabaron a través de motores de búsqueda tales como Google Académico y Google para garantizar el principio de actualidad y veracidad de la fuente. Se revisaron documentos y artículos científicos y de opinión, provenientes de páginas web, se realizó un comparativo de los datos recopilados, para compendiar y resaltar los de mayor importancia. 


\section{Resultados}

\section{La Administración del Siglo XXI}

Para (Melgar Callejas, s.f.), en la administración empresarial:

Se han adoptado nuevas filosofías, se han roto paradigmas, muchas técnicas y nuevos modelos están surgiendo, que prometen cambiar modernizar la Administración tanto Pública como Privada. La época de la comunicación nos permite en la actualidad ser más certeros en la solución de problemas que antes eran difíciles de entender. El uso de la inteligencia artificial en el futuro de la empresa, hace predecir que los Recursos Humanos como componente primordial tienen que especializarse para lograr avances significativos.

Los cambios son inevitables para la evolución de las empresas, sólo aquellos que adopten las nuevas herramientas competirán entre los mejores del mundo. Se avecina una nueva era, el siglo XXI con su principal avance, la tecnología, obliga al estudio del nuevo mercado global, con la finalidad de seleccionar y poner en uso aquellas herramientas que garanticen el alcance de las metas de la organización.

En este sentido, la administración del siglo XXI deberá ser tan flexible como los retos que se le presentarán; a continuación, se plasman los principales desafíos que las empresas enfrentarán con la finalidad de realizar un perfil del sistema administrativo del futuro.

\section{Retos}

- La Globalización.

La globalización es un proceso dinámico de creciente libertad e integración mundial de los mercados de trabajo, bienes, servicios, tecnología y capitales. Hoy en día las empresas deben de pensar en grande, porque es prácticamente inaudito seguir pensando en ser el mejor de una cuadra o un pueblo. Deben de pensar en ser el mejor del mundo, ya que la globalización ha reorientado los esfuerzos de las empresas a la calidad. Para poder competir no es necesario ser de un bloque específico, si no tener la capacidad para reinventarse, adaptarse y decidir. La globalización se orienta al cliente, olvida los monopolios y oligopolios para abrir los mercados y competir con todo el mundo, ahora puede operar a una empresa desde un ordenador portátil en la comodidad del hogar 
o a través de un GPS en el tránsito de alguna gran metrópoli. La globalización es la revolución que el mundo de los negocios esperaba. (Ramírez Velazco, 2012)

En este orden de ideas, el nuevo modelo de administración enfrenta muchos más desafíos, con la globalización se amplía el sistema organizacional, siendo más vulnerable ante la competencia mundial, resultando imperioso el aumento de calidad y la adopción de la automatización, sin descuidar el recurso humano como base de todos los procesos.

- La automatización de los procesos.

La automatización de procesos empresariales se refiere a la racionalización y optimización de procesos clave, que ayudan a las empresas a reducir los costos mediante la integración de soluciones tecnológicas. La automatización de procesos de negocio es un proyecto multifactorial. Por una parte, es un proyecto de negocio, después uno de tecnología y finalmente uno de recursos humanos. Esto, porque primeramente se definen los procesos de negocio a seguir en cada área, para seguido, automatizarlos con tecnología basada en hardware; comunicaciones y software que, necesariamente; serán ejecutados y administrados por personas encargadas de materializar los procesos dentro de la empresa. (Mendoza, 2018)

Hablar de tendencias para el futuro es casi imposible, por cuanto la tecnología avanza a pasos agigantados, mes a mes las tendencias varían, sin embargo, resulta interesante estudiar las tendencias actuales a efectos de establecer una proyección de lo que podría vislumbrarse en el siglo XXI para las organizaciones.

Según (Atalait, 2017) las tendencias para procesos empresariales se resumen de la siguiente manera:

A partir de que la tecnología comenzó a ofrecernos cada vez más formas y dispositivos para compartir información, las empresas se vieron inmersas en todo este proceso de movilización (procesos que se realizan a través de los dispositivos móviles) al cual se sumaron múltiples procesos internos de las organizaciones. Por otra parte, a partir de ahora y en el futuro se deben crear estrategias adecuadas a resguardar la información de las empresas. De esta forma es como se puede obtener seguridad a nivel global. Asimismo, los dispositivos externos desaparecieron como medio de resguardo de la información para poder acceder a los datos desde cualquier sitio. Por 
último, brindarles información a los colaboradores y herramientas para realizar los procesos es todo lo que se necesita para cumplir la mayoría de los objetivos.

Es fundamental para la administración el estudio de las nuevas tendencias en tecnología y los desafíos que implica su adopción a los fines de seleccionar la adecuada para su propósito y sacar el mayor provecho de esta herramienta informática.

- La preservación de los recursos naturales.

Es de vital importancia, el reto que representa para las empresas la conservación de los recursos naturales y con miras al futuro, cambiar el panorama actual en beneficio de la economía mundial y, por ende, la sustentabilidad de las empresas.

Al respeto, (Canal Clima, 2014) comenta:

La relación que se establece entre cambio climático y crisis económica no resulta sorpresiva. La extracción de recursos naturales es menester en muchas naciones y los resultados negativos ya pueden medirse en los balances financieros. El avance avasallador de empresas extractoras y distribuidoras de combustible no es casualidad en un modelo de desarrollo basado en los recursos fósiles, incluso ante la advertencia de su pronta caducidad y lo nocivo de su procesamiento para la naturaleza. La convergencia de todos estos elementos vincula de manera indisoluble al calentamiento global con la crisis económica mundial.

- El nuevo Paradigma de la Restructura de los Recursos Humanos.

Las nuevas corrientes requerirán de una radical estructuración de la Administración de Recursos Humanos, donde deje de ser una estructura horizontal, en que el administrador sea un facilitador, no un defensor de privilegios piramidales; que de gerente pase a ser un líder transformacional o sea que transforme el potencial, en realidad con liderazgo realidad con liderazgo multidimensional que esté presto a la eventualidad. Su actitud deberá ser de acción y no de reacción para provocar y crear las oportunidades y mantener siempre la organización en el tiempo y espacio, siempre firme y pujante. La tendencia es a inspirar para que dé lo mejor de sí y no a pensar, por lo tanto deberá dedicarse a grandes esfuerzos, ideas y analogías, clasificaciones y conceptos completamente nuevos, donde la imaginación y la creatividad será la clave para enfrentar el salto cuantitativo a que enfrenta la Administración de Recursos Humanos. (Melgar Callejas, s.f.) 
- Nuevos modelos de reingeniería.

(Manene, 2012) cita a Manganelli, cuando se refiere a la reingeniería como "el rediseño rápido y radical de los procesos estratégicos de valor agregado y de los sistemas, las políticas y las estructuras organizaciones que los sustentan, para optimizar los flujos del trabajo y la productividad de una organización". Asimismo, refiere que el concepto de reingeniería es una opción para reaccionar ante la situación actual y sus cambios. La reingeniería, enfocada en procesos eficientes que se basen en la satisfacción del cliente, logra eliminar la antigua forma de funcionamiento de las empresas.

Todos estos desafíos del siglo XXI se encuentran íntimamente relacionados, por ejemplo, es fundamental ante la adopción de nuevas tecnologías reorientar los procesos y los sistemas, esto lo cambia todo, como consecuencia, nuevos procesos, nuevas políticas, nueva estructura organizacional, es el modelo de empresa cambiante, de administración flexible, que saca provecho de la evolución del sistema organizacional y los diferentes elementos que la caracterizan.

- Constantes Bechmarking.

(Rojas Gutierrez, 2016) cita a la Comisión Directiva del International Benchmarking Clearinghouse, del American Productivity \& Quality Center, cuando se refiere al benchmarking como el "proceso de evaluación continuo y sistemático, un proceso mediante el cual se analizan y comparan permanentemente los procesos empresariales de una organización frente a los procesos de las compañías líderes en cualquier parte del mundo, a fin de obtener información que pueda ayudar a la organización a mejorar su performance y rendimiento".

Muchas organizaciones usan las técnicas de Benchmarking cuando quieren implementar un cambio radical en un determinado proceso altamente ligado a la consecución de estándares de calidad y mejores prácticas estimadas a escala global. Esto, sumado al ritmo de las innovaciones y mejoras permanentes en los procesos tecnológicos y de servicios, condiciona que el Benchmarking se constituya en una práctica de permanente evolución y alcances inacabados. (Manene, 2011)

- Desarrollo Organizacional.

(Angulo López, 2010) cita a Chiavenatto, cuando manifiesta que el desarrollo organizacional es un proceso planificado de modificaciones culturales y estructurales, que visualiza la 
institucionalización de una serie de tecnologías sociales, de tal manera que la organización quede habilitada para diagnosticar, planificar e implementar esas modificaciones con asistencia de un consultor. Es un esfuerzo educacional muy complejo, destinado a cambiar las actitudes, valores, los comportamientos y la estructura de la organización, de modo que esta pueda adaptarse mejor a las nuevas coyunturas, mercados, tecnologías, problemas y desafíos que surgen constantemente.

El desarrollo organizacional es un enfoque a largo plazo que no solo tiene el objetivo de incrementar el desempeño de la empresa, sino también mejorar la satisfacción de los empleados. Por un lado, el cambio sistemático permite a toda la empresa desempeñarse de una forma esperada. Por otro lado, trabaja con los empleados respecto de cómo pueden adaptarse a los cambios impuestos. El método es lo que facilita el cambio y el desarrollo en términos de tecnología, procesos y estructuras, mientras se tiene previsto la efectividad y la eficiencia de todo el negocio. En consecuencia, lidia con la mejora del desempeño y la salud generales de la empresa para producir mejores resultados; no necesariamente con un miembro individual. (Kruger Corporation, 2017)

Representa uno de los retos base de la reestructuración del modelo administrativo, ya que trata el recurso de mayor importancia de la empresa, su recurso humano, este fija las bases para que el resto de los cambios que requiera la organización estén bien asentados, se asienten con bases sólidas sobre la estructura principal de la organización.

- Auditoría administrativa.

La auditoría administrativa se puede considerar como un examen integral de la estructura de una organización, ya sea una empresa, institución o departamento gubernamental o cualquier otra entidad. También son evaluados los métodos de control, los medios de operación y el empleo de sus recursos humanos y materiales. Sin embargo, existen diferentes definiciones de auditoría administrativa de diversos expertos en el tema. (Conexionesan, 2017)

Entre los objetivos prioritarios para instrumentarla de manera consistente tenemos los siguientes: De control, destinados a orientar los esfuerzos en su aplicación y poder evaluar el comportamiento organizacional en relación con estándares preestablecidos. De productividad. Encauzan las acciones para optimizar el aprovechamiento de los recursos. De organización. Determinan que su curso apoye la definición de la estructura, competencia, funciones y procesos a través del manejo 
efectivo de la delegación de autoridad y el trabajo en equipo. De servicio. Representan la manera en que se puede constatar que la organización está inmersa en un proceso que la vincula cuantitativa y cualitativamente con las expectativas y satisfacción de sus clientes. De calidad. Disponen que tienda a elevar los niveles de actuación de la organización en todos sus contenidos y ámbitos. De cambio. La transforman en un instrumento que hace más permeable y receptiva a la organización. De aprendizaje. Permiten que se transforme en un mecanismo de aprendizaje institucional. De toma de decisiones. Traducen su puesta en práctica y resultados en un sólido instrumento de soporte al proceso de gestión de la organización. (Gerencie.com, 2017)

La revisión de los procesos implementados por la empresa, es fundamental para evaluar los resultados y mantener o adoptar las modificaciones necesarias para garantizar los mejores resultados.

\section{Características}

A continuación, se presenta un análisis de las diferentes características o inclinaciones hacia el futuro que la administración de empresas comienza a sufrir. Cabe destacar que las mismas surgen de los inminentes retos que confrontarán las empresas del futuro.

\section{- Demografía.}

(Villagrasa, 2012) cita a Peter Drucker, analista de economía y padre de la administración moderna, quien en base a las condiciones demográficas refiere:

Las nuevas condiciones demográficas van a producir enormes cambios en los próximos 25 años, especialmente en los países más desarrollados, ya que se prevé un envejecimiento relativo de la población. Esto traerá como consecuencia la imposibilidad de mantener los actuales sistemas previsionales, lo que obligará a seguir trabajando más años, aunque no en la forma actual de empleos a tiempo completo. Se prevé que las personas mayores de 50 años participen en el futuro de diferentes maneras, ya sea como temporeros, consultores, a tiempo parcial, entre otras. Se agregan, además, los cambios en el mercado, que actualmente está orientado a los jóvenes, cuando los compradores del futuro serán, principalmente, personas mayores. 
- La Nueva Fuerza de Trabajo.

Para Drucker, el conocimiento será el factor predominante en los años por venir. De hecho, ya lo es actualmente en gran manera, pero en la nueva sociedad será el principal recurso. Entre sus rasgos característicos se puede mencionar: que no tendrá fronteras; provocará además una gran movilidad social que permitirá el ascenso de los individuos con talento, que por otra parte será facilitado por el mejor acceso a la educación; que producirá fracasos y no solo éxitos, ya que muchos tendrán la capacidad y talento, pero no todos podrán conseguir lo mejor. Actualmente se entiende como trabajadores del conocimiento a personas muy preparadas en sus especialidades, con gran bagaje teórico; pero lo que se avecina para el futuro será también lo que se podría denominar tecnólogos del conocimiento. (Villagrasa, 2012)

- Los cambios de sector.

El siglo XXI verá el declinar relativo de la manufactura. El peso relativo de la manufactura está descendiendo en todos los países desarrollados, especialmente en EE UU, donde ha pasado de representar el $35 \%$ a mediados del siglo pasado, a menos de la mitad en la actualidad. En todos estos países, es el sector servicios el que ha crecido y ofrece al mercado laboral el mayor número de puestos de trabajo. (Villagrasa, 2012)

- Supervivencia de las corporaciones.

El futuro de las corporaciones también ocupa la atención de Drucker. Este fenómeno organizativo que aparece y se desarrolla en la primera mitad del siglo XX, ya ha sufrido una gran evolución en la segunda mitad de ese siglo. Las que inicialmente eran entidades nacionales, con algunas subsidiarias fuera, cada vez se fueron transformando en entidades organizadas globalmente según líneas de productos o servicios. Las multinacionales de 2025 se prevé que estarán unidas y controladas por estrategia, no por propiedad como hasta ahora. Seguirá habiendo propietarios, pero predominarán las alianzas, convenios de conocimiento, entre otros, que harán que se visualicen como bloques de confederaciones. Indudablemente requerirán un nuevo tipo de gerencia, distinto del actual. (Villagrasa, 2012) 
- Nueva estructura de los recursos humanos.

Otro aspecto que tendrá que tenerse en cuenta, en la nueva sociedad del conocimiento, es el trabajo con asociados, no subordinados, lo que obligará a replantearse toda la administración de personal, personal que además será predominantemente mayor, no joven. (Villagrasa, 2012)

Figura 1. Características de la administración del siglo XX Vs siglo XXI

\begin{tabular}{|l|l|l|}
\cline { 2 - 3 } \multicolumn{1}{l|}{} & \multicolumn{1}{c|}{ Siglo XX } & Siglo XI \\
\hline Trabajador & Manual & Tecnólogo del conocimiento \\
\hline Ubicación & Entidades nacionales & Entidades organizadas globalmente \\
\hline Cohesión & Propiedad individual & Alianzas estratégicas \\
\hline $\begin{array}{l}\text { Estructura de los } \\
\text { recursos humanos }\end{array}$ & Personal subordinado & Personal asociado \\
\hline Grupo etario & Joven & Predominantemente mayor \\
\hline $\begin{array}{l}\text { Sectores } \\
\text { predominantes }\end{array}$ & Manufactura & Servicios \\
\hline Mercados & Locales & Sin fronteras \\
\hline Compradores & Jóvenes & Principalmente mayores \\
\hline
\end{tabular}

Fuente: elaboración propia información tomada de (Villagrasa, 2012)

\section{Conclusiones}

La administración del siglo XXI debe ser tan flexible como los retos que se le avecinan, entre los principales e inminentes desafíos tenemos la globalización, fenómeno actual en creciente desarrollo que viene dado de la mano de los avances tecnológicos, lo que reorienta la administración a los procesos integrales, formándose una empresa que sale de su mercado nacional para abarcar el mercado mundial, adoptando como principal herramienta la tecnología. Precisamente, esta herramienta representa otro desafío, ya que su constante desarrollo obliga a las empresas a adoptar los medios informáticos disponibles para optimizar la calidad y rentabilidad de sus productos, la automatización de las empresas conlleva a la transformación de la administración, capaz de manejar las consecuencias de este reto, entre las principales, la evolución de la estructura de los recursos humanos, con la finalidad de sacar el mayor beneficio. 
La Preservación del ambiente es otro reto que enfrenta la administración del nuevo siglo, cada vez son más las empresas que se unen a la corriente ambientalista y es que no hay otra forma de percibir este beneficio común, en palabras sencillas, con un ambiente deteriorado o en el peor de los casos, sin planeta, no podremos sobrevivir, el administrador del siglo XXI debe concebir este concepto como básico para el desarrollo organizacional. Los nuevos modelos de reingeniería, benchmarking, desarrollo organizacional y auditoría administrativa, son igualmente fundamentales para el nuevo modelo de administración del siglo XXI.

En un sistema empresarial globalizado, con una súper población empresarial y mercados más amplios, es vital para las empresas que su administración se transforme e integre las nuevas herramientas tecnológicas que le permitan mantenerse en la competencia. Estar atento a los estudios de los desafíos del futuro, nuevos sistemas, nuevas políticas y todos aquellos cambios que requiera la organización, en un mundo donde los tiempos de acción son fundamentales para ganar terreno, sin dejar de lado el posicionamiento y la reinvención de productos y servicios. El comercio mundial obliga a las empresas a adaptarse a sus constantes cambios, por tanto es responsabilidad de la administración adoptar las nuevas características y tomar en cuenta los venideros desafíos a objeto de garantizar el alcance de las metas organizacionales.

\section{Referencias Bibliográficas}

Angulo López, E. (09 de Septiembre de 2010). Eumed.net. Recuperado el 13 de mayo de 2018, de http://www.eumed.net/tesis-doctorales/2012/eal/desarrollo_organizacional.html

Atalait. (06 de Septiembre de 2017). Atalait. Recuperado el 13 de mayo de 2018, de https://www.atalait.com/blog/automatizacion-de-procesos-empresariales

Canal Clima. (28 de Septiembre de 2014). Canal Clima. Recuperado el 13 de mayo de 2018, de http://www.canalclima.com/la-sobreexplotacion-de-recursos-naturales-genera-las-crisiseconomicas/

Chiavenato, I. (2004). Introducción a la Teoría General de la Administración (Séptima ed.). McGraw-Hill Interamericana. 
Conexionesan. (27 de Febrero de 2017). conexionesan. Recuperado el 13 de mayo de 2018, de https://www.esan.edu.pe/apuntes-empresariales/2017/02/la-auditoria-administrativa-una-accionindispensable/

Gerencie.com. (05 de Noviembre de 2017). Gerencie.com. Recuperado el 13 de mayo de 2018, de https://www.gerencie.com/auditoria-administrativa.html

Kruger Corporation. (22 de Febrero de 2017). Recuperado el 13 de mayo de 2018, de https://www.krugercorporation.com/blog-innovacion/desarrollo-organizacional/

Manene, L. M. (15 de Abril de 2011). luismiguelmanene. Recuperado el 13 de mayo de 2018, de http://www.luismiguelmanene.com/2011/04/15/benchmarkingdefiniciones-aplicaciones-tipos-yfases-del-proceso/

Manene, L. M. (23 de Enero de 2012). luismiguelmanene. Recuperado el 13 de mayo de 2018, de http://www.luismiguelmanene.com/2012/01/23/reingenieria-de-procesos-empresariales-y-sugestion-2/

Melgar Callejas, J. M. (s.f.). ufg.edu.sv. Recuperado el 12 de mayo de 2018, de http://www.ufg.edu.sv/ufg/theorethikos/Enero98/art11.html

Mendoza, A. (31 de Enero de 2018). GB Advosors. Recuperado el 13 de mayo de 2018, de http://www.gb-advisors.com/es/automatizacion-de-procesos-empresariales/

Ramírez Velazco, A. (08 de Noviembre de 2012). América Economía. Recuperado el 13 de mayo de 2018, de https://www.americaeconomia.com/negocios-industrias/la-globalizacion-y-elimpacto-en-el-mundo-empresarial

Rojas Gutierrez, K. (19 de Mayo de 2016). Gestiopolis. Recuperado el 13 de mayo de 2018, de https://www.gestiopolis.com/benchmarking-herramienta-desarrollo-empresarial/

Villagrasa, R. (05 de Diciembre de 2012). Koalasoft. Recuperado el 13 de mayo de 2018, de http://www.koala-soft.com/la-administracion-en-el-siglo-xxi

Villarreal, A. C. (29 de Octubre de 2013). Gestiopolis. Recuperado el 13 de mayo de 2018, de https://www.gestiopolis.com/historia-cambios-y-evolucion-de-la-administracion/ 